\title{
Interaction of SPIN90 with Dynamin I and Its Participation in Synaptic Vesicle Endocytosis
}

\author{
Yujin Kim, Sunyun Kim, Suho Lee, Sung Hyun Kim, Yoonju Kim, Zee Yong Park, Woo Keun Song, and Sunghoe Chang \\ Department of Life Science and Molecular Diseaome Research Center, Gwangju Institute of Science and Technology, Gwangju, South Korea 500-712
}

\begin{abstract}
SH3 protein interacting with Nck, $90 \mathrm{kDa}$ (SPIN90) is an Nck-binding protein that contains one Src homology 3 (SH3) domain, three proline-rich domains (PRDs), a serine/threonine-rich region, and a hydrophobic C-terminal region. Previously, we have shown that SPIN90 plays roles in the sarcomere assembly in cardiac muscles and in the formation of focal contacts in HeLa cells. Besides in heart, SPIN90 is also highly expressed in the brain, but its role in the neuronal system is completely unknown. Here, we found that SPIN90 is expressed in the presynaptic compartment in which it binds dynamin I, a key component of the endocytic machinery, and that it participates in synaptic vesicle endocytosis. Pull-down analysis and coimmunoprecipitation proved the associations of SPIN90 with dynamin I through SH3-PRD interaction. Overexpression of SPIN90 or knocking down SPIN90 by small interfering RNA impaired synaptic vesicle endocytosis. We further confirmed by the rescue experiments that the endocytic defects by SPIN90 expression come from its interaction with dynamin I. Exocytosis kinetics was not affected by SPIN90 expression. Together, our findings suggest that SPIN90 could modulate the interactions of dynamin I with other endocytic proteins that cooperate in the coated vesicle formation, thus regulating synaptic vesicle endocytosis.
\end{abstract}

Key words: SPIN90; dynamin I; synaptic vesicle; endocytosis; SH3; PRD

\section{Introduction}

SH3 protein interacting with Nck, $90 \mathrm{kDa}$ (SPIN90) is an Nckbinding protein that contains one Src homology 3 (SH3) domain, three Pro-rich motifs, and a serine/threonine-rich region (Lim et al., 2001). The SH3 domain of SPIN90 is very similar to that of WISH [a Wiskott-Aldrich syndrome protein (WASP)interacting $\mathrm{SH} 3$ protein] binding protein that is capable of enhancing neural WASP (N-WASP)-induced Arp2/3 complex activation, resulting in rapid actin polymerization (Rozelle et al., 2000; Rohatgi et al., 2001). SPIN90 is highly expressed in the heart, and it is known to participate in the sarcomere assembly during cardiac myocyte differentiation and to form a novel complex with $\beta$ PIX ( $\beta$-PAK-interacting exchange factor) and WASP for stable cell adhesion at focal contacts (Lim et al., 2001, 2003). Besides heart and skeletal muscle, the highest level of expression was also found in the brain, but its role in nerve system is presently unknown. One clue comes from the recent finding in that several accessory factors of vesicle endocytosis, including isoforms of dynamin, endophilin, and syndapin, have been detected in non-neuronal cells at sites of adhesion between cells and the

Received April 26, 2005; revised Aug. 27, 2005; accepted Aug. 29, 2005.

This research was supported by a grant from the National Research Laboratory (W.K.S.), by Korean Systems Biology Research Grant M10503010001-05N030100110 (Z.Y.P.), and by Brain Research Center of the 21st Century Frontier Research Program Grant M103KV010013 03 K2201 01320 (S.C.), funded by the Ministry of Science and Technology of the Republic of Korea. We thank Dr. James Rothman (Sloan-Kettering Cancer Institute, New York, NY) for providing the super-ecliptic synapto-pHluorin construct and Dr. Pietro De Camilli (Yale University, New Haven, (T) for providing the dynamin I constructs.

Correspondence should be addressed to Dr. Sunghoe Chang, Department of Life Science, Gwangju Institute of Science and Technology, 1 Oryong-dong Buk-gu, Gwangju, South Korea 500-712. E-mail: sunghoe@gist.ac.kr.

DOI:10.1523/JNEUROSCI.1643-05.2005

Copyright $\odot 2005$ Society for Neuroscience $\quad$ 0270-6474/05/259515-09\$15.00/0 substratum (Di Paolo et al., 2002; Lee and Camilli, 2002; Nikki et al., 2002; Wu et al., 2005). Thus, the possibility has been suggested that SPIN90 may have a role in the synapses, the cell adhesion counterpart in neurons.

Presynaptic nerve terminals recycle synaptic vesicle components through a local endocytic pathway. Clathrin-dependent endocytosis in nerve terminals is crucial for synaptic vesicle endocytosis (Slepnev and Camilli, 2000). Synaptic vesicle endocytosis involves various protein-protein interactions, and recent studies have revealed molecular details of this process. Dynamin is the GTPase that is targeted to the necks of endocytic coated pits in which it may function as an enzyme that pinches synaptic vesicles from the membrane leading to clathrin-coated vesicle formation at nerve terminals (Merrifield et al., 2002; Orth and McNiven, 2003). Dynamin contains a C-terminal proline-rich domain (PRD) that directly binds to the $\mathrm{SH} 3$ domains of multiple endocytic proteins, including amphiphysin (Takei et al., 1999), endophilin (Gad et al., 2000), and syndapin (Qualmann and Kelly, 2000). Therefore, it has been proposed that these molecules are linked together via their PRDs and their SH3 domains into protein machines to establish endocytic "hotspots" at presynaptic nerve terminals. In this study, we found that SPIN90 binds dynamin I through PRD-SH3 interaction, participating in synaptic vesicle endocytosis. We showed that the rate of synaptic vesicle endocytosis can be modulated by the availability of SPIN90 in the presynaptic compartments. The specificity of the interaction between SPIN90 with dynamin I in endocytosis regulation was confirmed by rescue experiments. Exocytosis kinetics was not affected by SPIN90 or SPIN90-SH3 expression. Thus, our findings proved that SPIN90 is one of the interacting partners of dynamin 
in presynaptic terminal, thus placing SPIN90 on synaptic vesicle endocytosis.

\section{Materials and Methods}

Glutathione S-transferase pull-down assays. To assess in vitro binding, a full-length insert of SPIN90 or its SH3-deletion mutant, SPIN90- $\Delta$ SH3, was subcloned into pRSET, a bacterial expression vector, and translated in vitro using the TNT T7-coupled reticulocyte lysate system. The radiolabeled products were incubated with purified glutathione $S$-transferase (GST) or GST fusion proteins (GST-dynamin I PRD) bound to glutathione beads. All incubations were performed in the binding buffer $[20 \mathrm{~mm}$ Tris, pH 8.0, 1 mм EDTA, $150 \mathrm{~mm} \mathrm{NaCl}, 0.2 \%$ Nonidet P-40, $1 \mathrm{~mm}$ phenylmethylsulfonyl fluoride (PMSF), $50 \mu \mathrm{g} / \mathrm{ml}$ aprotinin, $50 \mu \mathrm{g} / \mathrm{ml}$ leupeptin, and $50 \mu \mathrm{g} / \mathrm{ml}$ pepstatin] for $8 \mathrm{~h}$ at $4^{\circ} \mathrm{C}$. The glutathione beads were then washed four times in the binding buffer, and the radiolabeled proteins bound to the beads were solubilized by adding an SDS sample buffer in the presence of reducing agent and subjected to $8 \%$ SDS-PAGE. All reagents were purchased from Sigma (St. Louis, MO).

Cell culture and transfection. Embryonic day 18 primary rat hippocampal neurons were prepared as described previously (Chang and Camilli, 2001). Briefly, hippocampi were dissected from embryonic day 18 Sprague Dawley fetal rats, dissociated with papain, and triturated with a polished half-bore Pasteur pipette. Approximately 150,000 cells in MEM supplemented with $0.6 \%$ glucose, $1 \mathrm{~mm}$ pyruvate, $2 \mathrm{~mm}$ L-glutamine, $10 \% \mathrm{FBS}$, and antibiotics were plated on poly-D-lysine-coated glass coverslips in a $60 \mathrm{~mm}$ Petri dish. Four hours after plating, the medium was replaced with culture medium consisting of Neurobasal medium (Invitrogen, Gaithersburg, MD), B-27, and 0.5 mm L-glutamine.

Neurons were transfected using the calcium-phosphate method (Chang and Camilli, 2001) and then used $2 \mathrm{~d}$ after transfection. SPIN90green fluorescent protein (GFP) was transfected at $7 \mathrm{~d}$ in vitro (DIV) to examine the effect on the actin cytoskeleton, and synaptopHluorin ( $\mathrm{spH}$ ) (kindly provided by Dr. James Rothman, Sloan-Kettering Cancer Institute, New York, NY) alone or with full-length SPIN90, SPIN90- $\Delta$ SH3, or SPIN90-SH3 tagged with hemagglutinin (HA) was transfected at 10 DIV for endocytosis assays. Cotransfection of $\mathrm{spH}$ and SPIN90 constructs was performed in the ratio of 1:2 to 1:5. After the endocytosis assays, the cells were fixed and stained with anti-GFP and anti-HA antibodies to confirm cotransfection. The amount of $\mathrm{spH}$ construct transfected was fixed for constant fluorescent signals.

Immunoblot analysis. Approximately 2,000,000 of hippocampal neurons plated on $100 \mathrm{~mm}$ tissue culture dishes coated with poly-D-lysine and grown for $3 \mathrm{~d}, 7 \mathrm{~d}$, or 2 weeks were lysed in a lysis buffer (1\% SDS, 1 mm sodium orthovanadate, $10 \mathrm{~mm} \mathrm{NaF}, 10 \mathrm{~mm}$ Tris- $\mathrm{HCl}, \mathrm{pH}$ 7.4, $1 \mathrm{~mm}$ PMSF, $10 \mathrm{~mm}$ leupeptin, $1.5 \mathrm{~mm}$ pepstatin, and $1 \mathrm{~mm}$ aprotinin). Cell lysates were scratched out immediately, boiled $5 \mathrm{~min}$, and clarified by centrifugation at $12,000 \times g$ for $10 \mathrm{~min}$. Protein concentrations were measured using a bicinchoninic acid Protein Assay Reagent kit (Pierce, Rockford, IL). Constant amounts of proteins were separated on SDSPAGE and transferred to polyvinylidene difluoride membranes (BioRad, Hercules, CA). The membranes were then blocked for $1 \mathrm{~h}$ with $5 \%$ nonfat dry milk in $10 \mathrm{~mm}$ Tris- $\mathrm{HCl}, \mathrm{pH} 7.5,100 \mathrm{~mm} \mathrm{NaCl}$, and $0.1 \%$ Tween 20, after which they were incubated with the respective primary antibodies, anti-SPIN90 or anti-dynamin I (Affinity BioReagents, Golden, $\mathrm{CO}$ ), and then with horseradish peroxidase-conjugated antirabbit IgG (Jackson ImmunoResearch, West Grove, PA). The antigenantibody complexes were detected with the enhanced chemiluminescence reagents (Amersham Biosciences, Piscataway, NJ). Blots were stripped by heating to $60^{\circ} \mathrm{C}$ for $30 \mathrm{~min}$ in a stripping buffer $(100 \mathrm{~mm}$ $\beta$-mercaptoethanol, 2\% SDS, and $62.5 \mathrm{~mm}$ Tris- $\mathrm{HCl}, \mathrm{pH}$ 6.7) and reprobed with anti- $\alpha$-tubulin (Sigma) as a control.

Coimmunoprecipitation. Cells were washed twice with cold PBS and extracted for $1 \mathrm{~h}$ at $4^{\circ} \mathrm{C}$ in a modified radioimmunoprecipitation assay buffer (50 mм Tris-HCl, pH 7.5, 5 mм EDTA, 150 mm NaCl, 1\% NP-40, $1 \mathrm{~mm}$ sodium orthovanadate, $1 \mathrm{~mm}$ PMSF, $10 \mathrm{~mm}$ leupeptin, $1.5 \mathrm{~mm}$ pepstatin, and $1 \mathrm{~mm}$ aprotinin). The extracts were then clarified by centrifugation at $12,000 \times g$ for $10 \mathrm{~min}$, and the protein concentration in the supernatants were determined using Bradford Protein Assay Reagent kit (Bio-Rad). Samples containing $1 \mathrm{mg}$ of total protein were then taken for subsequent immunoprecipitation for $14 \mathrm{~h}$, followed by an additional $4 \mathrm{~h}$ of incubation at $4^{\circ} \mathrm{C}$ with protein A-Sepharose beads. The immunoprecipitates were extensively washed with the lysis buffer and then subjected to SDS-PAGE and immunoblot analysis.

In-gel digestion and peptide sample preparation. All solvents used in this procedure were HPLC grade. The SDS-polyacrylamide gels were stained with Coomassie brilliant blue. Protein bands were excised from the stained gel. Then the excised bands were washed three times with 1:1 (v/v) solution of acetonitrile/deionized water for $10 \mathrm{~min}$ and dehydrated with $100 \%$ acetonitrile. The bands were finally washed with $1: 1(\mathrm{v} / \mathrm{v})$ solution of $100 \%$ acetonitrile/ $100 \mathrm{~mm}$ ammonium bicarbonate and dried using a Speed-Vac. Proteins contained in the gel pieces were reduced by using $10 \mathrm{~mm}$ tris(2-carboxyethyl)phosphine in $0.1 \mathrm{M}$ ammonium bicarbonate at $56^{\circ} \mathrm{C}$ for $45 \mathrm{~min}$ and then alkylated with $55 \mathrm{~mm}$ iodoacetamide in $0.1 \mathrm{M}$ ammonium bicarbonate at room temperature for $30 \mathrm{~min}$. Next, the washing step above was repeated on the alkylated sample. After the washing step, the gel pieces were dried and soaked in sequencing-grade trypsin solution $(500 \mathrm{ng}$ ) on ice for $45 \mathrm{~min}$. Then the gel pieces were immersed in $100 \mu \mathrm{l}$ of $50 \mathrm{~mm}$ ammonium bicarbonate, $\mathrm{pH} 8.0$, at $37^{\circ} \mathrm{C}$ for $14-18 \mathrm{~h}$. The resulting peptides were extracted sequentially for 20 min with $45 \%$ acetonitrile in $20 \mathrm{~mm}$ ammonium bicarbonate, $45 \%$ acetonitrile in $0.5 \%$ trifluoroacetic acid (TFA), and $75 \%$ acetonitrile in $0.25 \%$ TFA with agitation. The extracts containing tryptic peptides were pulled together and evaporated under vacuum.

Micro liquid chromatography-mass spectrometry/mass spectrometry analysis and protein database search. In-gel digested proteins were loaded onto fused silica capillary columns $(100 \mu \mathrm{m}$ inner diameter, $360 \mu \mathrm{m}$ outer diameter) containing $8 \mathrm{~cm}$ of $5 \mu \mathrm{m}$ particle size Aqua $\mathrm{C}_{18}$ reversephase column material. The column was placed inline with an Agilent Technologies (Palo Alto, CA) HP 1100 quaternary liquid chromatography (LC) pump and a splitter system was used to achieve a flow rate of $250 \mathrm{nl} / \mathrm{min}$. Buffer A ( $5 \%$ acetonitrile and $0.1 \%$ formic acid) and buffer B ( $80 \%$ acetonitrile and $0.1 \%$ formic acid) were used to make a $90 \mathrm{~min}$ gradient. The gradient profile started with 5 min of $100 \%$ buffer A, followed by a 60 min gradient from 0 to $55 \%$ buffer B, a 25 min gradient from 55 to $100 \%$ buffer B, and a 5 min gradient of $100 \%$ buffer B. Eluted peptides were directly electrosprayed into an LTQ linear ion trap mass spectrometer (ThermoFinnigan, Palo Alto, CA) by applying $2.3 \mathrm{kV}$ of direct current voltage. A data-dependent scan consisting of one full mass spectrometry (MS) scan (400-1400 mass/charge) and five datadependent MS/MS scans were used to generate MS/MS spectra of eluted peptides. Normalized collision energy of $35 \%$ was used throughout the data acquisition. MS/MS spectra were searched against the National Center for Biotechnology Information rat protein sequence database using Bioworks version 3.1 (Beckman Coulter, Fullerton, CA) and Sequest Cluster System (14 nodes; Thermo Electron, San Jose, CA). DTASelect was used to filter the search results and the following Xcorr values were applied to different charge states of peptides: 1.8 for singly charged peptides, 2.2 for doubly charged peptides, and 3.2 for triply charged peptides. Manual assignments of fragment ions in each MS/MS spectra were performed to confirm the protein database search results.

Immunocytochemistry. Immunocytochemistry was performed as described previously (Chang and Camilli, 2001). Briefly, cells were fixed in $4 \%$ formaldehyde $/ 4 \%$ sucrose/PBS for $15 \mathrm{~min}$ and permeabilized for 5 $\min$ in $0.25 \%$ Triton X-100/PBS. They were blocked for $30 \mathrm{~min}$ in $10 \%$ $\mathrm{BSA} / \mathrm{PBS}$ at $37^{\circ} \mathrm{C}$ and incubated in primary antibodies $/ 3 \% \mathrm{BSA} / \mathrm{PBS}$ for $2 \mathrm{~h}$ at $37^{\circ} \mathrm{C}$ or overnight at $4^{\circ} \mathrm{C}$ and then washed six times for 2 min each in $\mathrm{PBS}$, followed by an additional incubation for $45 \mathrm{~min}$ at $37^{\circ} \mathrm{C}$ in secondary antibodies/3\% BSA/PBS. Coverslips were mounted on a slide glass, and fluorescence images were acquired on a Zeiss (Thornwood, NY) Axiovert 35 inverted microscope with a $40 \times, 1.4$ numerical aperture (NA) oil lens using a CoolSNAP-Hq CCD camera (Roper Scientific, Tucson, AZ) driven by IPLab (Scanalytics, Fairfax, VA) imaging software. Primary antibodies used are as follows: anti-dephospho-tau-1 (Chemicon, Temecula, CA), antimicrotubule-associated protein (MAP2) (Sigma), anti-GFP (Abcam, Cambridge, UK), anti-HA (Roche, Penzberg, Germany), anti-Myc (Upstate Biotechnology, Lake Placid, NY), anti-FLAG (Sigma), and anti-dynamin I (Affinity BioReagents) antibodies. Secondary antibodies were obtained from Jackson Immunoresearch. 
SynaptopHluorin endocytosis/exocytosis assay. Hippocampal neurons 12-14 DIV transfected with synaptopHluorin were mounted in a custom-built stimulation chamber on the stage of an Olympus Optical (Tokyo, Japan) IX-71 inverted microscope with a $40 \times, 1.0$ NA or a $60 \times$, 1.4 NA oil lens. Cells were continuously perfused at room temperature $\left(\sim 24^{\circ} \mathrm{C}\right)$ with Tyrode's solution containing $119 \mathrm{~mm} \mathrm{NaCl}, 2.5 \mathrm{~mm} \mathrm{KCl}, 2$ mм $\mathrm{CaCl}_{2}, 2 \mathrm{~mm} \mathrm{MgCl}_{2}, 25 \mathrm{~mm}$ HEPES, buffered to $\mathrm{pH} 7.4,30 \mathrm{~mm}$ glucose, $10 \mu \mathrm{M}$ 6-cyano-7-nitroquinoxaline-2,3-dione, and $50 \mu \mathrm{M}$ D,L-2amino-5-phosphonovaleric acid. Time-lapse images were acquired every $10 \mathrm{~s}$ for 6 min using a CoolSNAP-ES CCD camera (Roper Scientific) driven by MetaMorph Imaging software (Universal Imaging Corporation, West Chester, PA) with a GFP optimized filter set (Omega Optical, Brattleboro, VT). From the fourth frame, cells were stimulated $(1 \mathrm{~ms}$, $20-50 \mathrm{~V}$, bipolar) for $15 \mathrm{~s}$ at $20 \mathrm{~Hz}$ using a Grass SD9 stimulator (GrassTelefactor, West Warwick, RI). Quantitative measurements of the fluorescence intensity at individual boutons were obtained by averaging a selected area of pixel intensities using MetaMorph software. Light from a mercury lamp was shuttered using a VMM1 Unibilitz shutter (Vincent Associates, Rochester, NY). Net fluorescence changes were obtained by subtracting average of the intensities of the first four frames $\left(F_{0}\right)$ from the intensity of each frame $\left(F_{\mathrm{t}}\right)$ for individual boutons. Then they were normalized to the maximum fluorescence intensity $\left(F_{\max }-F_{0}\right)$ and averaged. The decay of fluorescence was fitted with a single exponential. All fitting procedures were done using individual error bias to weight the fit using SigmaPlot 6.0 (Systat Software, Point Richmond, CA). In some experiments in which fluorescence decay deviated from a singleexponential behavior, we obtained the best-fitting single-exponential function from the early portion of decay. Data are presented as mean \pm SEM. For rescue experiments, SPIN90-Myc was coexpressed with dynamin I-HA or N-WASP-FLAG, and SPIN90-SH3-Myc was coexpressed with dynamin I-PRD-HA. For exocytosis assay, control neurons expressed spH or neurons expressed SPIN90-HA or SPIN90-SH3-HA with $\mathrm{spH}$ were preincubated with bafilomycin A1 for $60 \mathrm{~s}$ and stimulated for $120 \mathrm{~s}$ at $10 \mathrm{~Hz}$. Net fluorescence changes were obtained by subtracting average of the intensities of the first three frames $\left(F_{0}\right)$ from the intensity of each frame $\left(F_{\mathrm{t}}\right)$ for individual boutons, and then they were normalized to the maximum fluorescence intensity $\left(F_{\max }-F_{0}\right)$ and averaged. Bafilomycin A1 (CalBiochem, San Diego, CA) was dissolved in DMSO as 0.2 $\mathrm{mm}$ and diluted to a final concentration of $0.5 \mu \mathrm{m}$ before experiments. Bafilomycin was applied throughout fluorescent measurements. Expression of each construct was confirmed by the retrospective immunostainings using specific antibodies (mouse anti-Myc, rabbit anti-FLAG, and rat anti-HA antibodies), and only immunopositive neurons were included in the analysis.

RNA interference. Small interfering (si) RNAs of SPIN90 were designed on the basis of rat SPIN90 cDNA sequence (GenBank accession number XP_23855), targeting to the region of nucleotides 1767-1787 (siRNA-1) and 1771-1791 (siRNA-2). A pair of $66 \mathrm{nt}$ complementary oligonucleotides were synthesized separately with additions of an Acc65I site at the $5^{\prime}$ end and a HindIII site at the $3^{\prime}$ end. The forward primer sequences were 5'-GTACCTCGGACGATCCTGTGCGTATCTTTCAAGAGAAGATACGCACAGGATCGTCCTTTTTGGAAA-3' (siRNA-1) and 5'-GTACCTCGATCCTGTGCGTATCTTCAGATCAAGAGTCTGAAGATACGCACAGGATCTTTTTGGAAA-3' (siRNA-2); the underlined letters represent the SPIN90 siRNA sequence. The annealed $66 \mathrm{bp}$ cDNA fragment with SPIN90 siRNA was cloned into the Acc65IHindIII sites of the psiRNA-hH1GFPzeo G2 vector (InvivoGen, San Diego, CA). The efficiency of siRNAs was tested in Rat-1 cell, a rat fibroblast origin, and then confirmed in neurons. Hippocampal neurons were transfected with SPIN90-specific siRNAs using calcium phosphate and analyzed by immunoblot, immunofluorescence, and endocytosis assay.

FM5-95 endocytosis assay. $\mathrm{N}$-(3-trimethylammoniumpropyl)-4-(6(4-(diethylamino)phenyl)hexatrienyl)pyridinium dibromide (FM5-95) was used at a concentration of $15 \mu \mathrm{M}$ in Tyrode's solution. Pools of synaptic vesicles were labeled by electrical stimulation for $30 \mathrm{~s}$ at $10 \mathrm{~Hz}$ in the presence of FM5-95. After 10 min of washing in dye-free Tyrode's solution, the image was taken and neurons were stimulated for $2 \mathrm{~min}$ at $10 \mathrm{~Hz}$ to unload FM5-95. A fully unloaded image was taken afterward.
A

$\mathrm{SH} 3 \mathrm{M} / \mathrm{S}$ 120 SPIN90(1-722)

\section{SH3: SH3 domain : Proline Rich Domain(PRD)}

B

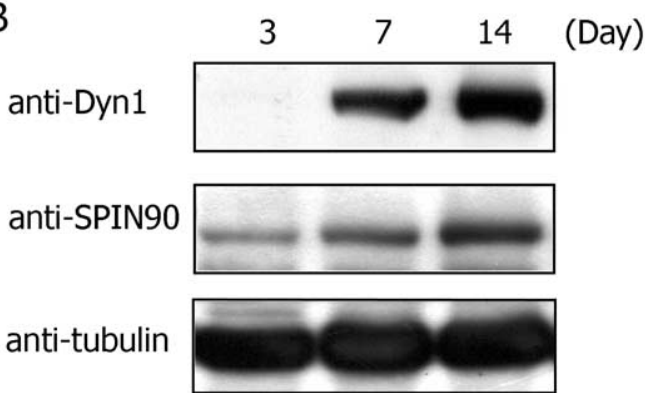

Figure 1. The expression of SPIN90 in hippocampal neurons in culture increases as the neurons mature. $A$, Schematic diagram of SPIN90 B B, Cultured hippocampal neurons at 3, 7, and 14 DIV were lysed and immunoblotted with a specific SPIN90 antibody. Unlike dynamin I (Dyn1), the expression of SPIN90 was observed in the early neurons at 3 DIV. The expression of both dynamin I and SPIN90 increased as the neurons matured.

Net fluorescence changes were obtained by subtracting the intensity of the unloaded image from the intensity of the loaded image. A 15 min rest period was inserted between the end of the first unloading stimulus train and the start of the second loading stimulus. Images were acquired using a CoolSNAP-ES CCD camera driven by MetaMorph Imaging software with a FM4-64 [N-(3-triethylammoniumpropyl)-4-(6-(4-diethylamino)phenyl)hexatrienyl)pyridinium dibromide] optimized filter set. Statistics were performed using SigmaStat (Systat Software). Data are presented as mean \pm SEM.

\section{Results}

SPIN90 is expressed in rat hippocampal neurons in culture

Cultured rat hippocampal neurons, a well established system for neuronal differentiation, start to form synapses $\sim 7 \mathrm{~d}$ in culture and differentiate into functionally mature neurons within $14 \mathrm{~d}$. To examine whether SPIN90 is expressed in the hippocampal neurons in culture, a Western blot analysis was performed using a specific SPIN90 antibody. Previously, the highest levels of expression were found in the brain, the heart, and the skeletal muscle (Lim et al., 2001). Consistently, we found that SPIN90 is expressed in hippocampal neurons, and its expression increased as the neurons matured (Fig. 1). We also found that SPIN90 is similarly expressed in the cortical neurons in culture (data not shown).

\section{SPIN90 is found at presynaptic terminals of hippocampal neurons}

To find the localization of endogenous SPIN90 in neurons, we performed immunocytochemistry using an SPIN90-specific antibody. Although more diffusely present, the axonal SPIN90 along the processes formed many puncta that colocalized with the presynaptic vesicle maker synaptophysin (Fig. 2A-C). Similar results were obtained using two other synaptic vesicle markers, synaptobrevin 2 and synaptotagmin, indicating that SPIN90 highly expresses in presynaptic terminals (Fig. 2D-I). When we focused our observation on the dendritic SPIN90 staining, immunoreactivity was frequently found juxtaposed to presynaptic vesicle markers, indicating the expression of SPIN90 in both presynaptic and postsynaptic compartments. 


\section{SPIN90 binds dynamin I via its $\mathrm{SH} 3$} domain in vitro and in vivo

Recently, a number of SH3 domaincontaining proteins that interact with the PRD of dynamin have been identified, and these interactions have been implicated in clathrin-mediated endocytosis (Shupliakov et al., 1997; Simpson et al., 1999). SPIN90 also contains an SH3 domain, and its presence at presynaptic nerve terminals strongly suggests that SPIN90 may bind dynamin through PRD-SH3 interaction and participate in synaptic vesicle endocytosis. To test this hypothesis, we first performed micro LC-MS/MS analysis using GST-SPIN90-SH3 (Fig. 3A). GSTendophilin-SH3 was used as a positive control because it has been shown to interact with dynamin I preferentially (Ringstad et al., 1999). Seven bands were clearly identified in SDS-PAGE gel, and, after subsequent in-gel digestion, micro LCMS/MS, and protein database search, the protein corresponding to each band was identified. Among seven proteins, dynamin I and N-WASP were known proteins, and another five proteins were either unknown or not clearly identified yet (Fig. $3 A$ ). Although the binding of SPIN90-SH3 with dynamin I is not as strong as that of endophilin-SH3, it is clear that dynamin I is the major binding partner of SPIN90SH3. The association of SPIN90 with dynamin was further investigated. A GST pull-down analysis was conducted with in vitro-translated SPIN90. Figure $3 B$ shows that SPIN90 coprecipitated with GSTdynamin I-PRD. An SPIN90 mutantdeleted $\mathrm{SH} 3$ domain (SPIN90- $\Delta \mathrm{SH} 3$ ) failed to bind GST-dynamin I-PRD, indicating that SPIN90 interacts with dynamin I via its $\mathrm{SH} 3$ domain. The subse-

quent coimmunoprecipitation analysis using cultured rat hippocampal neuron lysates confirmed that SPIN90 indeed associates with dynamin I in vivo (Fig. 3C). Thus, SPIN90 binds with the dynamin I-PRD in an SH3 domain-dependent and direct interaction. The interaction of SPIN90 with dynamin I was further confirmed by immunocytochemistry. Neurons were stimulated by high $\mathrm{KCl}$ before fixation to recruit dynamin I to endocytic sites, and they were double stained with SPIN90 antibody and dynamin I antibody. Although SPIN90 showed rather diffused cytosolic staining in an unstimulated condition, under a stimulated condition, SPIN90 and dynamin I almost perfectly colocalized with each other throughout the neurites (Fig. 3E). Indeed, high $\mathrm{KCl}$ stimulation has resulted in the increase of binding dynamin I with SPIN90 (Fig. 3D).

\section{Overexpression of SPIN90 impairs clathrin-mediated synaptic vesicle endocytosis}

We next investigated whether the interaction of SPIN90 with dynamin I could be involved in synaptic vesicle endocytosis. To measure synaptic vesicle endocytosis in cultured hippocampal neurons, $\mathrm{spH}$ was used as an assay system. $\mathrm{spH}$ is vesicle-
B

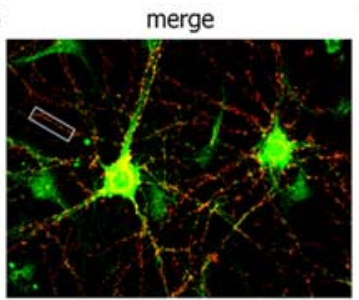

C anti-synaptophysin
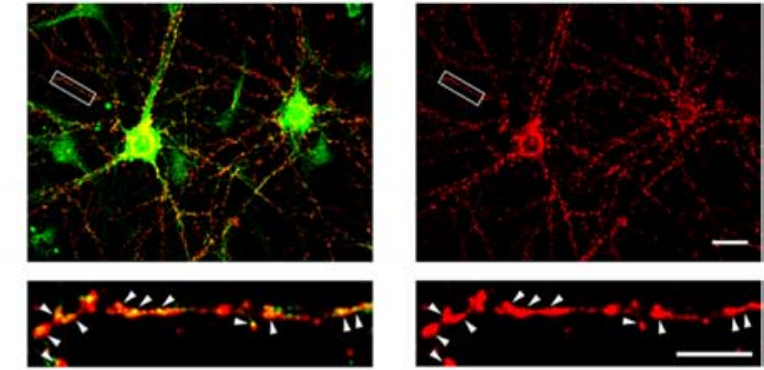

$\mathrm{E}$
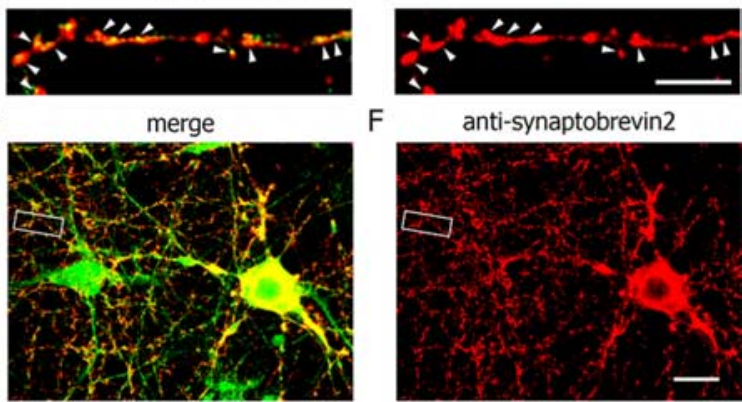

$\mathrm{F}$
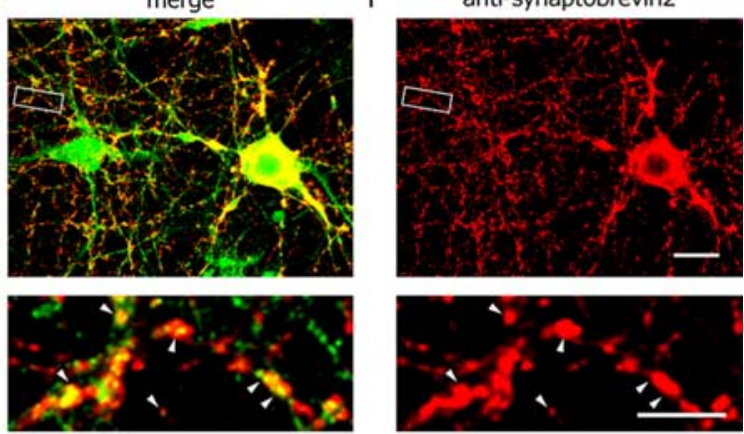

merge

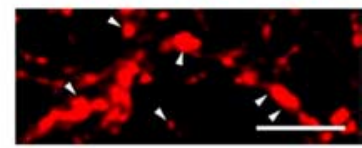

$\mathrm{H}$

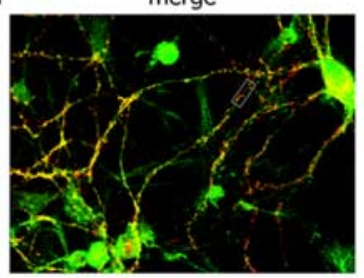

I
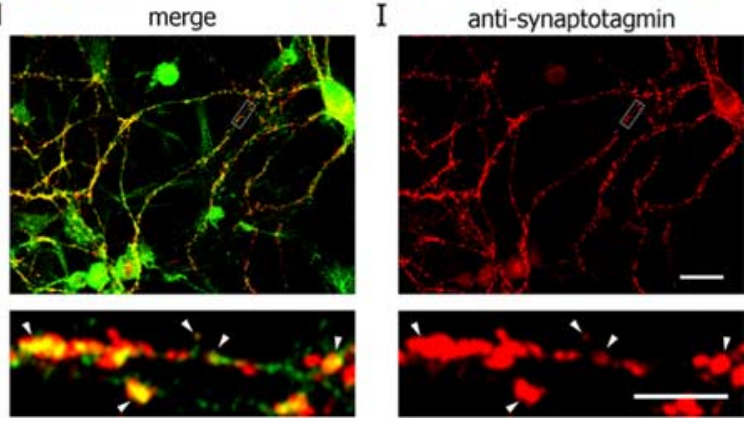

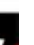

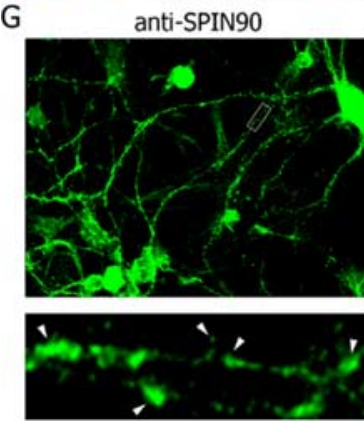

14 DIV were fixed, permeabilized, and immunostained with anti-SPIN90 antibody $(\boldsymbol{A}, \boldsymbol{D}, \boldsymbol{G})$ and anti-synaptophysin $(\boldsymbol{C})$, anti-synaptobrevin2 $(\boldsymbol{F})$, and antisynaptotagmin $(\boldsymbol{I})$, respectively. $\boldsymbol{B}, \boldsymbol{E}, \boldsymbol{H}$, Middle panels are the merged images of immunostainings of SPIN90 and respective presynaptic vesicle markers. High-magnification view of the region enclosed by a rectangle is shown below each image. Arrowheads indicate the regions in which colocalizations are readily seen. Note that most regions in which presynaptic markers exist are also positive in SPIN90 immunoreactivity. Scale bars: low magnification, $20 \mu \mathrm{m}$; high magnification, $5 \mu \mathrm{m}$.

associated membrane protein-2 (VAMP-2)/synaptobrevin-2 fused with a variant of GFP, which is sensitive to $\mathrm{pH}$ change (ecliptic pHluorin). The fluorescence of ecliptic pHluorin is quenched once synaptic vesicles are endocytosed and reacidified (from external $\mathrm{pH} \sim 7.4$ to an internal $\mathrm{pH}$ of synaptic vesicle $\sim 5.5$ ). It has been proven that reacidification occurs rapidly during endocytosis and that the fluorescence change of $\mathrm{spH}$ would thus reflect endocytosis. Moreover, $\mathrm{spH}$ expression does not perturb the normal physiology of hippocampal synaptic terminals. Thus, $\mathrm{spH}$ has been reliably used in previous studies to measure synaptic vesicle recycling in real time at individual presynaptic boutons (Sankaranarayanan and Ryan, 2000).

When introduced in hippocampal neurons, $\mathrm{spH}$ was localized in the regions of tau-1-positive neurites but not in the regions of MAP2-positive neurites, implying that $\mathrm{spH}$ appears preferentially in axonal compartments like endogenous VAMP2 (supplemental Fig. 1, available at www.jneurosci.org as supplemental material). $\mathrm{spH}$ was enriched in varicosities, which were also stained by FM4-64, a marker for functional presynaptic terminals. When electrical stimulation [300 action potentials (APs)/20 $\mathrm{Hz}$ ] was applied, the fluorescence intensity of individual $\mathrm{spH}$ 


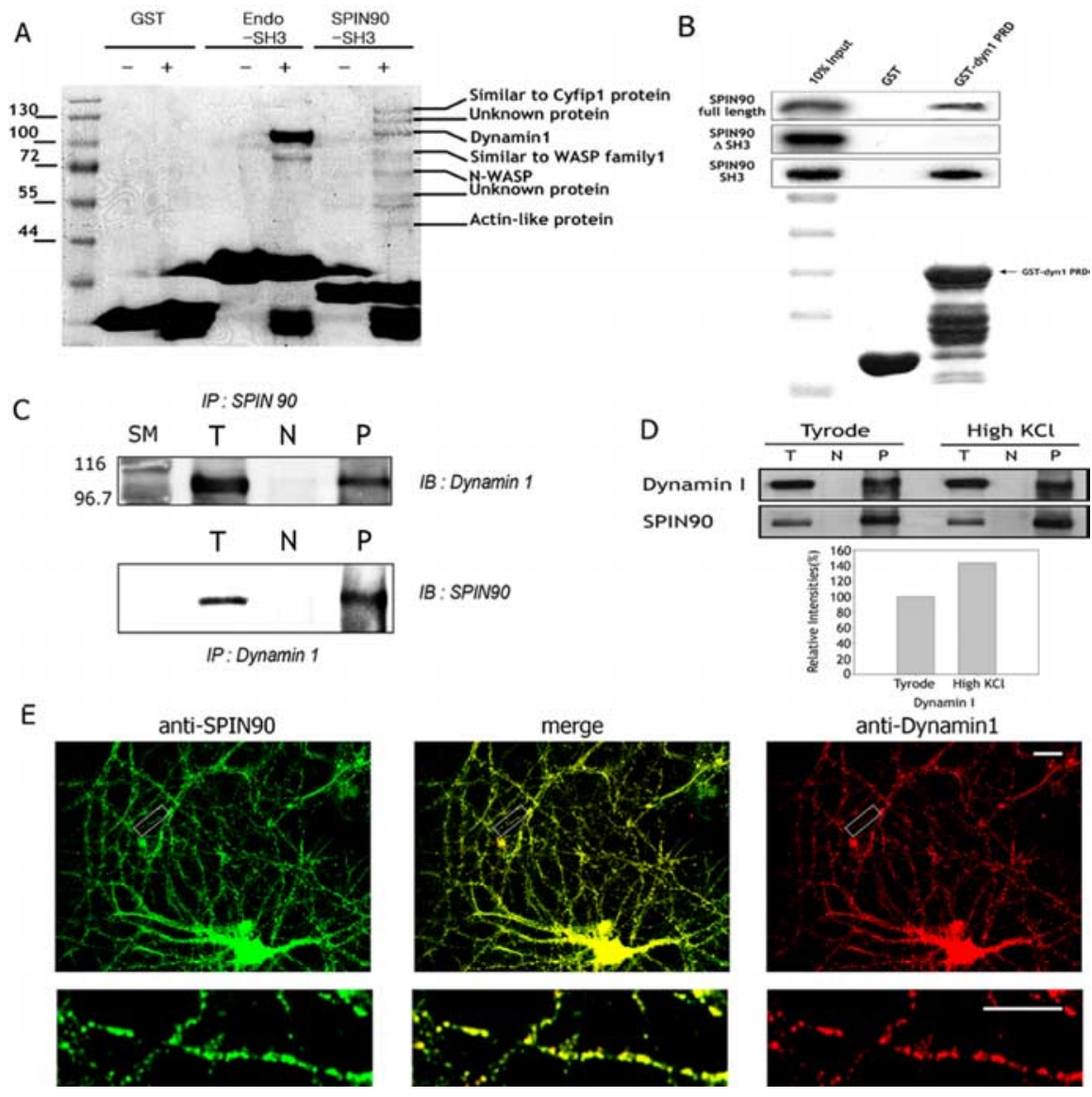

Figure 3. SPIN90 binds dynamin I via its SH3 domain in vitro and in vivo. A, Brain lysates were incubated with GST, GSTendophilin-SH3, and GST-SPIN90-SH3, and the SDS-polyacrylamide gels were stained with Coomassie brilliant blue. Protein bands were excised from the stained gel and analyzed by micro LC-MS/MS (for a detailed description, see Materials and Methods). Seven bands appeared, and each protein was identified with a protein database search. Note that, although the association of SPIN90 with dynamin I is not as strong as endophilin, among known proteins thus far, dynamin I and N-WASP are the major binding partner of SPIN90-SH3. B, ${ }^{35}$ S-Met labeled, in vitro-translated SPIN90 full-length or SPIN90- $\Delta$ SH3 was incubated with purified GST-dynamin I PRD or GST protein immobilized on glutathione-agarose beads, after which GST pull-down assays were performed. Bound proteins were analyzed by SDS-PAGE and visualized by autoradiography. C, To confirm the association of SPIN90 and dynamin I, in vivo, hippocampal neuron lysates were immunoprecipitated with anti-SPIN90 antibody or anti-dynamin I antibody and immunoblotted (IB) with anti-dynamin I or anti-SPIN90 antibody, respectively. SM, Size marker; T, total cell lysate; N, normal rabbit serum; $P$, precipitant. $\boldsymbol{D}$, High $\mathrm{KCl}$ stimulation increases the association of dynamin I with SPIN90. Relative intensity of dynamin I was increased $\sim 46 \%$ with high $\mathrm{KCl}$ stimulation over the Tyrode's solution. T, Total cell lysate; N, normal rabbit serum; P, precipitant. $\boldsymbol{E}$, Hippocampal neurons at 14 DIV were stimulated with $60 \mathrm{~mm}$ high $\mathrm{KCl}$ solution to recruit dynamin I to endocytic sites and were fixed immediately, permeabilized, and immunostained with anti-SPIN90 antibody and anti-dynamin I antibody. The second antibodies used are Oregon green-conjugated anti-mouse lgG for SPIN90 and Texas Red-conjugated anti-rabbit lgG for dynamin I. Middle panels are the merged images of immunostainings of SPIN90 and dynamin I. High-magnification views are of the regions enclosed in rectangles, respectively. Note the perfect colocalization of SPIN90 staining with dynamin I immunoreactivity. Scale bars: low magnification, $20 \mu \mathrm{m}$; high magnification, $5 \mu \mathrm{m}$.

boutons increased rapidly and reached a peak that decayed after stimulation. The fluorescence decay corresponded to internalization of $\mathrm{spH}$, i.e., synaptic vesicle endocytosis.

To investigate the effect of SPIN90 on endocytosis, SPIN90-HA was cotransfected with $\mathrm{spH}$ into hippocampal neurons. Most of the neurons $(>90 \%)$ that expressed $\mathrm{spH}$ also expressed SPIN90-HA, which was confirmed by retrospective immunostaining of the $\mathrm{HA}$ at the end of each experiment. The HA-negative neurons were not included in the analysis. During a train of 300 action potentials stimulation, the fluorescence intensities of the boutons expressing $\mathrm{spH}$ with or without SPIN90 increased and subsequently decayed with an exponential time course after stimulation. The kinetics of synaptic vesicle endocytosis as monitored by $\mathrm{spH}$ fluorescence decay was significantly slowed in boutons expressing SPIN90 compared with non-expressing boutons ( $\tau=106.4 \pm 9.7$ for SPIN90 expression; $\tau=76.4 \pm 4.4$ for control) (Fig. $4 A, C$ ). Indeed, SPIN90-expressing synapses retained a significantly higher fraction of residual spH fluorescence $200 \mathrm{~s}$ after stimulation ( $26 \pm 0.02 \%$ for SPIN90 expression vs $0.05 \pm 0.02 \%$ for control) (Fig. $4 H$ ), reflecting a slower rate of endocytosis compared with control boutons. The effect of SPIN90 to slow the rate of endocytosis is readily apparent in a comparison of 1/tau with and without SPIN90 expression. We plotted histograms for the rates of $\mathrm{spH}$ fluorescence decay (measured as the reciprocal of the time constant) from individual puncta from a number of separate experiments, both with and without SPIN90 coexpression (Fig. 4D,E). SPIN90 expression increased the number of boutons in that $\mathrm{spH}$ fluorescence decayed slowly and shifted the distribution of rates to lower endocytosis rates.

Because SPIN90-dynamin interactions seemed to be important for endocytosis, an endocytosis assay was performed to examine whether its $\mathrm{SH} 3$ domain is responsible for the inhibition of synaptic vesicle endocytosis caused by SPIN90 overexpression. The SH3 domain of SPIN90 (SPIN90-SH3) tagged with HA was introduced with $\mathrm{spH}$ into neurons instead of the full-length SPIN90, and an endocytosis assay was performed. As expected, cells expressing SPIN90-SH3 showed slow kinetics of endocytosis $(\tau=100.1 \pm 11.1)$ and retained a fraction of residual $\mathrm{spH}$ fluorescence comparable with those expressing a full-length SPIN90 (Fig. 4B, C,H). To confirm the effect of $\mathrm{SH} 3$ domain of SPIN90, we expressed the SH3 deletion mutant of SPIN90 (SPIN90- $\Delta$ SH3) with $\mathrm{spH}$ and measured the endocytosis kinetics. Expression of an SPIN90- $\Delta \mathrm{SH} 3 \mathrm{had}$ no effect on endocytosis, and the time course of endocytosis of SPIN90- $\Delta$ SH3expressing boutons was not significantly different from that of control boutons (Fig. $4 B, C$ ). Histograms for the rates of $\mathrm{spH}$ fluorescence decay also proved that the $\mathrm{SH} 3$ domain of SPIN90 is responsible for the slowing effect on endocytosis by SPIN90 (Fig. $4 D-G$ ).

\section{Endocytic defect comes from interaction of SPIN90 with dynamin I}

Previously, we found that SPIN90 binds WASP through SH3PRD interaction (Lim et al., 2003). Indeed, WASP is the only other protein that is known to bind SPIN90 through SH3-PRD interaction so far. Depletion of endogenous N-WASP by sequestering it to mitochondria or by introducing anti-N-WASP antibodies impaired endocytosis (Kessels and Qualmann, 2002). Because both N-WASP and dynamin I bind SPIN90 through SH3-PRD interaction, the endocytosis defects that we have de- 
tected in Figure 4 could have been resulted from the interaction of SPIN90 with $\mathrm{N}$-WASP rather than with dynamin I. To rule out this possibility, we performed the rescue experiment using co-overexpression of dynamin I with SPIN90. Figure 5 showed that co-overexpression of dynamin I with SPIN90 successively rescued the endocytic defects by the overexpression of SPIN90 alone, suggesting that endocytic impairment by SPIN90 expression is attributable to its interaction with dynamin I $(\tau=76.75 \pm 4.9 \mathrm{~s}$ for control; $89.86 \pm 5.6$ s for SPIN90/dynamin I coexpression) (Fig. $5 A, B$ ). Endocytic defect by SPIN90-SH3 expression was also rescued by co-overexpression with dynamin-PRD (Fig. 5B). Coexpression of N-WASP with SPIN90, however, failed to rescue endocytic defects by SPIN90 alone (117.84 \pm $9.2 \mathrm{~s}$ for SPIN90/N-WASP coexpression), further confirming the specificity of the interaction between dynamin I and SPIN90 (Fig. $5 A, B$ ), and this interaction is responsible for endocytic regulation by SPIN90.

\section{Synaptic vesicle exocytosis is not} affected by SPIN90 overexpression In the presynaptic nerve terminal, endocytosis is tightly coupled to exocytosis. To find whether SPIN90 has an effect on synaptic vesicle exocytosis, we measured the rate of exocytosis during SPIN90 expression using $\mathrm{spH}$ with bafilomycin A1 (Fernandez-Alfonso and Ryan, 2004). Bafilomycin A1, a V-type ATPase inhibitor, blocks acidification of recently endocytosed vesicles and traps them in an alkaline state during recycling. Blocking reacidification provides a way to count the number of exocytotic events, independent of the speed of endocytosis. The rate of exocytosis at each condition was determined by measuring the slope of the $\mathrm{spH}$ responses in the presence of bafilomycin during a 300 AP stimulus. Figure 6 showed that the kinetics of synaptic vesicle exocytosis was not affected by SPIN90 expression (the slope from linear fits to the data, 0.022 for control and 0.025 for SPIN90; $p>0.7)$, suggesting that the role of SPIN90 in synaptic vesicle recycling resides in the endocytic pathway. Exocytosis rate was also comparable between SPIN90-SH3expressing boutons and control boutons (data not shown).

\section{Synaptic vesicle endocytosis during the stimulation is not affected by SPIN90 overexpression}

Because synaptic vesicle endocytosis occurs not only after the stimulation but also during the stimulation, we tested whether SPIN90 also affects the rate of endocytosis during a train of action potentials. Because the fluorescence change in the presence of bafilomycin reflects the exocytosis while in the absence of bafilomycin, the fluores-
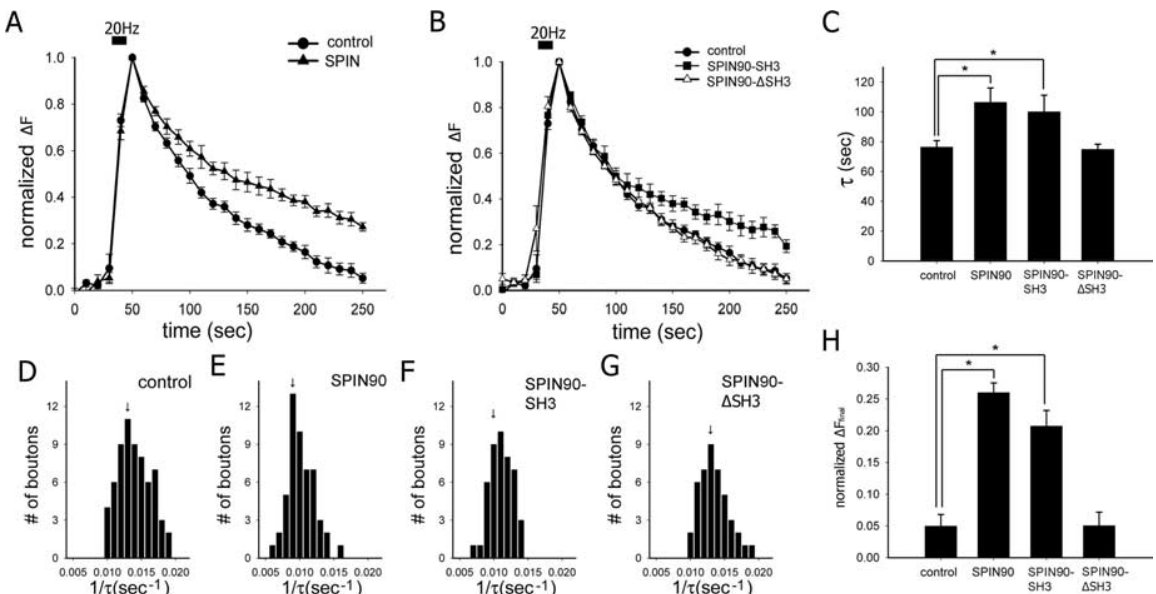

Figure 4. SPIN90 overexpression impairs clathrin-mediated synaptic vesicle endocytosis. $\boldsymbol{A}$, Average fluorescence intensity profiles, plotted as $\Delta F / F_{0}$ against time, after a stimulation with 300 action potentials at $20 \mathrm{~Hz}$ (dark bar). The decay of fluorescence was fitted by a single exponential, with $\tau=76.4 \pm 4.4(n=11)$ for control and $\tau=106.4 \pm 9.7(n=7)$ for SPIN90-expressing boutons $(\boldsymbol{A}, \boldsymbol{C})$. B . Time course of fluorescence intensity after a stimulation with 300 action potentials at $20 \mathrm{~Hz}$ (dark bar) SPIN90-SH3; $\tau=74.9 \pm 3.5, n=13$ for SPIN90- $\Delta$ SH3). Note that there is no significant difference in decay kinetics between control boutons and SPIN90- $\Delta$ SH3-expressing boutons $(\boldsymbol{B}, \boldsymbol{C}) . \boldsymbol{D}-\mathbf{G}$, Frequency histograms of the reciprocal of spH fluorescence SPIN90 full length $(\boldsymbol{E} ; n=51)$, SPIN90-SH3 $(\boldsymbol{F} ; n=45)$, and SPIN90- $\Delta$ SH3 $(\boldsymbol{G} ; n=43)$. $\boldsymbol{H}$, Residual spH fluorescence values malized to the initial resting state of each presynaptic bouton, $200 \mathrm{~s}$ after stimulation. Note that SPIN90- and SPIN90-SH3expressing synapses retained a significantly higher fraction of residual spH fluorescence $200 \mathrm{~s}$ after stimulation. ${ }^{*} p<0.01$, significantly different from the control value, Student's $t$ test.
A

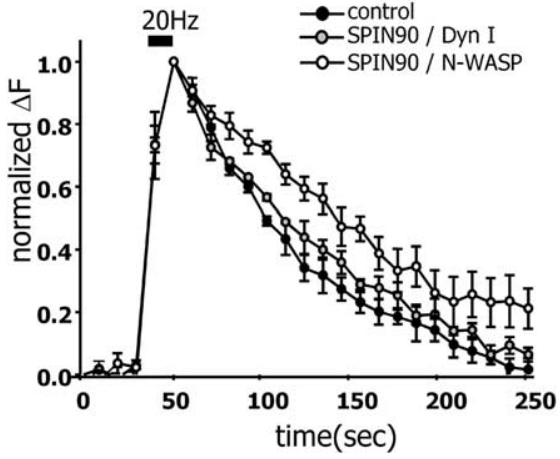

B

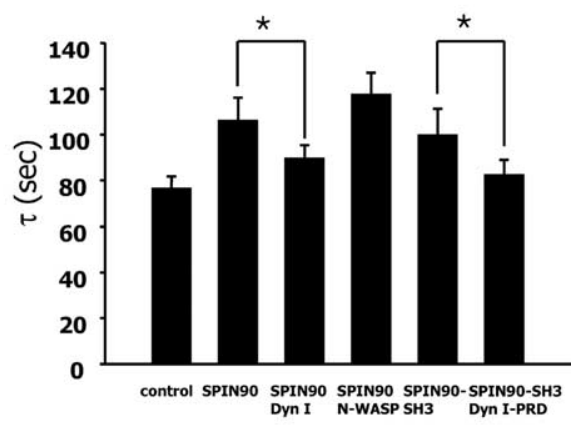

Figure 5. Endocytic defect comes from interaction of SPIN90 with dynamin I. Average fluorescence intensity profiles, plotted as $\Delta F / F_{0}$ against time, after a stimulation with 300 action potentials at $20 \mathrm{~Hz}$ (dark bar). $\boldsymbol{A}, \boldsymbol{B}$, The decay of fluorescence was fitted by a single exponential with $\tau=89.86 \pm 5.65(n=20)$ for SPIN90/dynamin I (Dyn I) coexpression, $\tau=117.84 \pm 9.2(n=12)$ for SPIN90/N-WASP coexpression, and $\tau=83.28 \pm 6.21(n=14)$ for SPIN90-SH3/dynamin I-PRD coexpression. Coexpression of /dynamin I and SPIN90-SH3/dynamin I PRD successfully rescue endocytic defects by SPIN90 or SPIN90-SH3, respectively, whereas there is no significant difference in decay kinetics between SPIN90 boutons and SPIN90/N-WASP-expressing boutons. ${ }^{*} p<0.01$, significantly different from the control value, Student's $t$ test.

cence changes represent the balance of exocytosis and endocytosis, and we could calculate the time course of endocytosis during the stimulation by simply subtracting the $\mathrm{spH}$ fluorescence values.

As shown in Figure 7, the difference provides the time course of endocytosis and slope obtained by linear fits to the $300 \mathrm{AP}$ train was compared with that for the time course of exocytosis. The result showed that endocytosis/exocytosis ratio with SPIN90 overexpression was not significantly different from that of control ( 0.4 for control; 0.37 for SPIN90 overexpression; $p>1$, Student's $t$ test; $n=29$ boutons for control, $n=31$ boutons for SPIN90), indicating that, although SPIN90 overexpression im- 


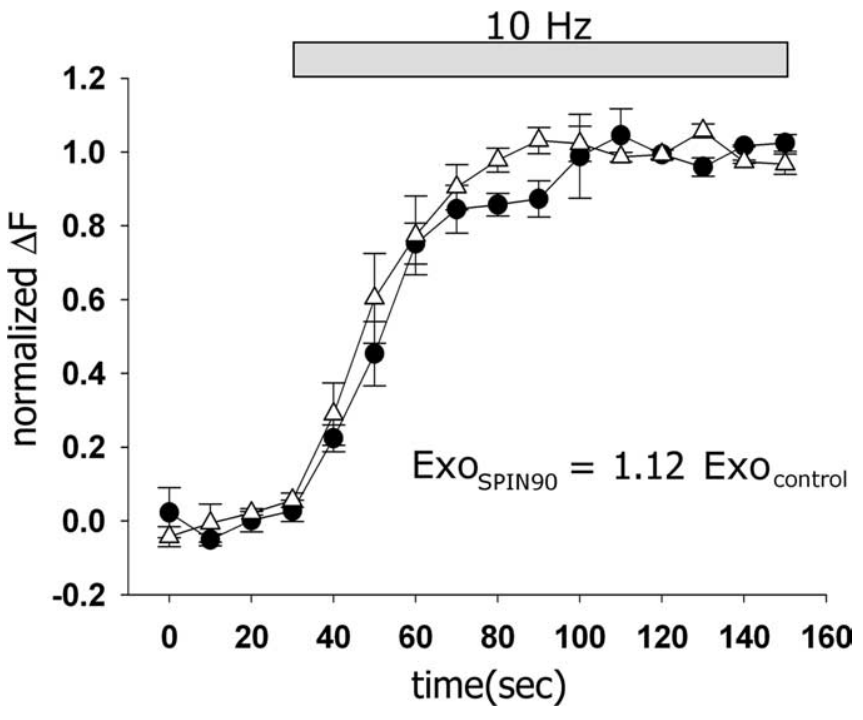

Figure 6. Exocytosis kinetics was not affected by SPIN90 expression. Control neurons expressing spH or neurons expressing SPIN90-HA with spH were preincubated with bafilomycin A1 $(0.5 \mu \mathrm{M})$ for $60 \mathrm{~s}$ and stimulated for $120 \mathrm{~s}$ at $10 \mathrm{~Hz}$ in the presence of bafilomycin $\mathrm{A} 1$ to exclusively measure exocytosis. The fluorescence values were normalized to the peak fluorescence change for each experimental condition. The rate of exocytosis at each condition was obtained from linear fits to the data during a $300 \mathrm{AP}$ stimulus (the slope, 0.022 for control and 0.025 for SPIN90), and the ratio of the slope values (1.12) was indicated. Note that exocytosis kinetics at each condition was not significantly different from each other. $p>0.7, t$ test; $n=$ 52. $\mathrm{ExO}_{\text {SPIN90 }}$ and $\mathrm{ExO}_{\text {control, }}$ The rate of exocytosis in SPIN90-expressing neurons and in control neurons, respectively.

pairs synaptic vesicle endocytosis, it does not affect the endocytosis during a train of action potentials.

\section{Knock-down of endogenous SPIN90 using siRNA impairs synaptic vesicle endocytosis}

We next investigated the effect of knocking down endogenous SPIN90 on synaptic vesicle endocytosis. We have used two independent siRNA constructs and suppression of SPIN90 expression by each siRNA in Rat-1 fibroblasts was confirmed by immunoblotting, whereas expression of tubulin was shown to be unaffected (Fig. 8C,D). The efficiency of each siRNA was further confirmed in hippocampal neurons (supplemental Fig. 2, available at www.jneurosci.org as supplemental material). Although the SPIN90 protein level seems to be reduced to $\sim 80 \%$ of control level by immunoblotting, considering the low transfection efficiency in primary cultured neurons (1-5\%), conservative estimation tells us that the expression of SPIN90 would be reduced to $<5-10 \%$ of control level in siRNA-transfected neurons. This is confirmed by immunofluorescence staining of SPIN90 in siRNAtransfected neurons, in which SPIN90 immunoreactivity is almost abolished (Fig. 8E).

Because the siRNA construct we used is tagged GFP, we were not able to use $\mathrm{spH}$ as an assay system. Instead, to measure the rate of endocytosis, FM5-95, a red-shift fluorescent lipophilic dye, was used. After the first loading and unloading, the second loading was performed with $20 \mathrm{~s}$ delay after the electrical stimulation began. In the $20 \mathrm{~s}$ before the onset of FM5-95 application, some vesicles would undergo endocytosis, escape the labeling, and thus remain unlabeled. The slower the endocytosis, the higher the intensity of FM5-95 staining will be (Micheva et al., 2003). In such experiments, the FM5-95 intensity of siRNAtransfected synaptic boutons was invariably higher than the intensity of control boutons $(62.9 \pm 3.07$ for control, $n=22 ; 88.3 \pm 3.69$ for
siRNA-1, $n=25 ; 92.2 \pm 3.15$ for siRNA-2, $n=20 ; 90.7 \pm 4.39$ for SPIN90 overexpression, $n=29 ; p<0.01$ ) (Fig. $8 B$ ). This strongly indicates that endocytosis proceeds more slowly when endogenous SPIN90 is reduced by siRNA treatment, thus demonstrating that the reduction of endogenous SPIN90 by siRNA causes a severe synaptic vesicle endocytosis defect in neurons.

\section{Discussion}

SPIN90 was first identified as an Nck-binding protein, and it has been suggested that SPIN90 plays a role in the regulation of actin cytoskeleton through its interaction with various actin regulating proteins. In this study, we report that SPIN90 binds dynamin I, a key component of the endocytic machinery, through PRD-SH3 interaction, and that SPIN90 is involved in synaptic vesicle endocytosis. Our experiments revealed that SPIN90 is highly expressed in presynaptic and postsynaptic compartments. Pulldown analysis using in vitro-translated SPIN90 with GSTdynamin I-PRD proved the interaction of SPIN90 with dynamin I. Coimmunoprecipitation of dynamin I with SPIN90 and colocalization of SPIN90 with dynamin I in primary cultured neurons indicate that SPIN90 associates with dynamin I in vivo, and that it may play a role in synaptic vesicle endocytosis. Indeed, overexpression of SPIN90 or knocking down endogenous SPIN90 by siRNAs impaired synaptic vesicle endocytosis in neurons, and this effect was caused by the interaction of the SH3 of SPIN90 with dynamin I-PRD. This is further confirmed by the rescue experiment using SPIN90/dynamin I coexpression.

SPIN90 has high degree of similarity to VacA-interacting protein, $54 \mathrm{kDa}$ (VIP54), AF3p21 (ALL-1 fused gene from chromosome $3 \mathrm{p} 21$ ), and Diaphanous protein interacting protein [Dia interacting protein-1 (DIP-1)]. Indeed, the N-terminal half of these proteins seems identical, although the C-terminal half shows splicing variant pattern. VIP54 colocalizes with vimentinand desmin-containing intermediate filaments in human parietal cells (Bernard et al., 2000), whereas Sano et al. (2000) identified the gene $A F 3 p 21$ as a mixed lineage leukemia (MLL) fusion partner gene whose product is expressed as a fusion protein with MLL. DIP-1 is known to regulate actin polymerization and cell adhesion turnover downstream of the Rho-mDia pathway (Satoh and Tominaga, 2001).

Tau value of endocytosis in control neuron in our experiments seems to be longer compared with previously reported values ( $\sim 65$ s) (Li and Murthy, 2001). Previous studies have used an neuron-astrocyte mix culture system, and it has now been well established that glial cells secrete various growth-enhancing factors that promote neuronal development as well as synaptic functions (Pfrieger and Barres, 1997). Because SPIN90 is also expressed in glial cells, we wanted to bypass any possible complication from glial cells and to have near-pure neuron cultures instead of neuron-astrocytes coculture. To this purpose, we have used serum-free medium with AraC treatment (see Materials and Methods). Thus, the difference in the tau value may come from different culture systems, especially without involvement of glial cell-derived factors.

Synaptic vesicle endocytosis involves the interaction of multiple proteins at different steps. Dynamin, a key player of endocytosis, has been proposed to function as a mechanochemical enzyme that pinches vesicles from the plasma membrane, although its precise role in endocytosis is still controversial (Qualmann et al., 2000; Orth and McNiven, 2003). Recent studies suggested that dynamin may activate downstream mediators of endocytosis rather than playing a role as a mechanochemical en- 
zyme (Schafer, 2002). One such mediator is actin, and a connection of dynamin to actin function is of significant interest. The mechanism by which dynamin affects actin dynamics during endocytosis is unclear, but some clues have emerged. Recent studies place dynamin on actin comets generated either by Listeria or by type I phosphatidylinositol 5-phosphatate kinase (Lee and Camilli, 2002). Evanescent field microscopic study showed that the inward movement of vesicle occurred immediately after a brief burst of dynamin recruitment, and that it was accompanied by transient actin assembly (Merrifield et al., 2002). Endocytic vesicles may use actin polymerization to move into the cytosol after being pinched off from the plasma membrane (Lee and Camilli, 2002; Merrifield et al., 2002). Several proteins that bind with dynamin can also interact with actin or with proteins that regulate actin assembly. These include profilin (Witke et al., 1998), Abp1 (Kessels et al., 2001), cortactin (McNiven et al., 2000), syndapin (Qualmann and Kelly, 2000), intersectin (Hussain et al., 2001), Grb2 (Miki et al., 1994), and Nck (Benesch et al., 2002). Interestingly, all of these proteins contain the $\mathrm{SH} 3$ domain that interacts with the PRD of dynamin. Thus, it has been suggested that the PRD of dynamin is a critical determinant for the interaction of this protein with the actin cytoskeleton and thus links endocytosis to the actin polymerization. Our results showed that SPIN90 is one of the interacting partners of dynamin in neurons and regulates synaptic vesicle endocytosis. The specificity of the interaction in endocytosis between SPIN90 with dynamin I was confirmed by a series of rescue experiments. Because SPIN90 binds WASP and it contains putative binding sites for actin-regulating proteins in C-terminal regions, it is possible that SPIN90 may play a role as a linker between the actin dynamics and the synaptic vesicle endocytosis, although this needs additional investigation.

Our results showed that SPIN90 does not affect the rate of endocytosis during the stimulation, although the endocytosis after stimulation (post-endocytosis) was impaired (Fig. 7). This could be result from the mechanistic difference between the endocytosis during the stimulation and post-endocytosis. Indeed, it has been shown previously in many studies that, during intense firing of action potentials, synaptic vesicles do not undergo full fusion but rather form brief fusion pores, thus releasing neurotransmitters through the "kiss-and-run" mode, followed by
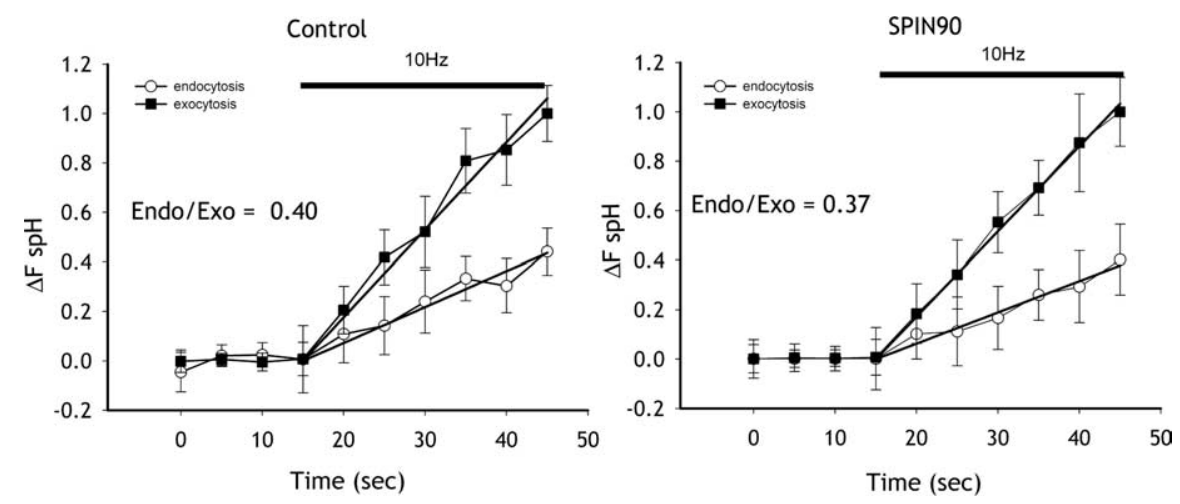

Figure 7. The rate of endocytosis during the stimulation was not affected by SPIN90 expression. The graphs indicate the rate of endocytosis (End) and exocytosis (Exo) during stimulation with $300 \mathrm{AP}$ at $10 \mathrm{~Hz}$. Endocytosis during the stimulus was derived by subtracting the spH fluorescence in the absence of bafilomycin from that in the presence of bafilomycin for each bouton. The fluorescence values were normalized to the peak fluorescence change in the presence of bafilomycin. The ratios of the slopes were obtained by linear fits to the $300 \mathrm{AP}$ train. Although endocytosis/exocytosis ratio with SPIN90 overexpression was smaller than that of control ( 0.4 for control and 0.37 for SPIN90 overexpression), it was not statistically significant. $p>1$, Student's test; $n=$ 29 boutons for control, $n=31$ boutons for SPIN90.

\section{A}

Condition 1

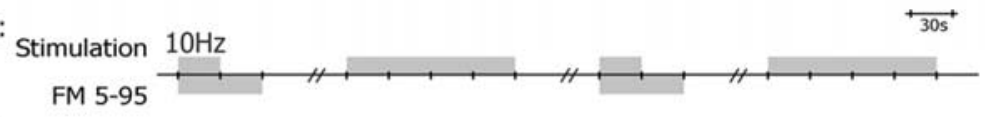

Condition 2:
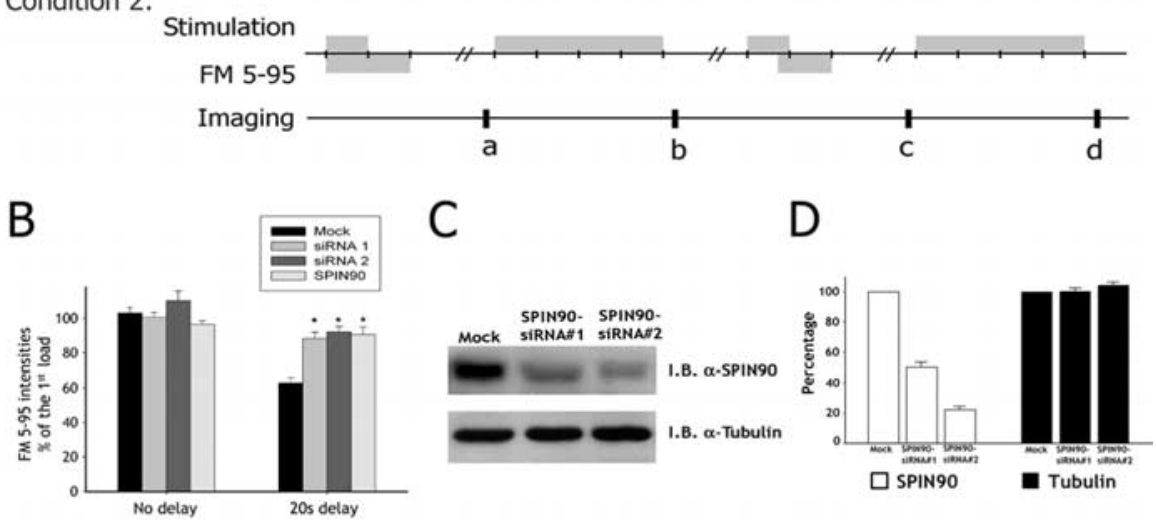

E
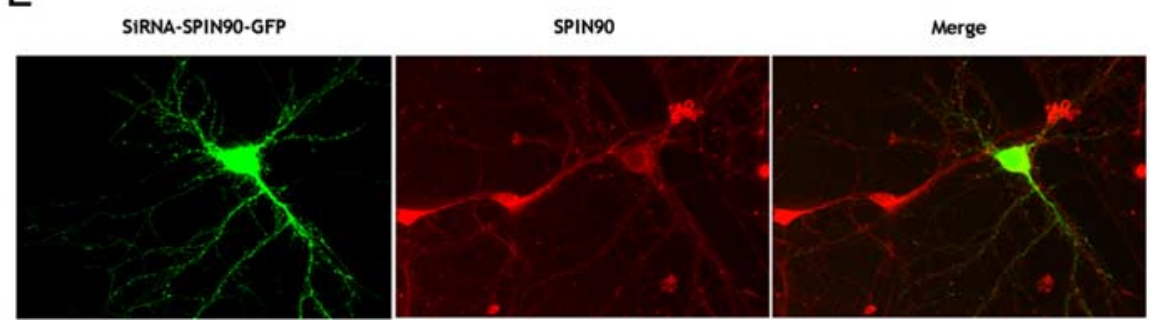

Figure 8. Knock-down of endogenous SPIN90 results in slower endocytosis. A, Summary of the loading- unloading protocol used to compare endocytosis in neuronal cultures treated with siRNA with that in control cultures. Neurons were treated with GFP-tagged SPIN90-specific siRNAs or vector only (Mock), and two consecutive load- unload cycles with FM5-95 were performed. For control condition 1, the second load of FM5-95 is identical to the first one without delay. For condition 2, dye added $20 \mathrm{~s}$ after the onset of stimulation in the second load. $\boldsymbol{B}$, FM5-95 loading intensities were expressed as percentage of the intensity of the first load and averaged across the synaptic population. In the control condition, compared with the first load, both mock- and siRNAtransfected boutons contained comparable amounts of FM5-95 intensities in the second load ( $103.1 \pm 3.25$ for control, $n=22$; $100.7 \pm 2.75$ for siRNA-1, $n=25 ; 110.3 \pm 5.53$ for siRNA-2, $n=20 ; 96.4 \pm 2.09$ for SPIN90 overexpression, $n=29$ ). In condition 2, however, siRNA-treated boutons showed a significantly higher FM5-95 intensity compared with mock-treated ones $(62.9 \pm 3.07$ for control, $n=22 ; 88.3 \pm 3.69$ for siRNA-1, $n=25 ; 92.2 \pm 3.15$ for siRNA-2, $n=20 ; 90.7 \pm 4.39$ for SPIN90 overexpression, $n=29 ; p<0.01$ ), indicating slower endocytosis in siRNA-treated boutons. $\boldsymbol{C}, \boldsymbol{D}$, Rat- 1 fibroblasts were transfected with GFP-tagged SPIN90-specific siRNA, after which the lysates were immunoblotted with anti-SPIN90 antibody. Relative band intensity was measured and displayed as histograms. E, Representative images of SPIN90 knock-down by siRNA-2. Hippocampal neurons that have been transfected with SPIN90 siRNA were fixed, permeabilized, and immunostained with SPIN90 antibody. Note that SPIN90 immunoreactivity in the siRNA-transfected neuron was mostly abolished compared with that in the neighboring neuron. 
rapid reuse (Pyle et al., 2000; Harata et al., 2001; Zakharenko et al., 2002). Thus, the rapid retrieval without full fusion of synaptic vesicles possibly does not involve conventional machinery of slow recycling, such as the interactions of dynamin with other endocytic proteins.

Because SPIN90 contains three proline-rich motifs as well as the $\mathrm{N}$-terminal $\mathrm{SH} 3$ domain, this protein could play a role as a multi-scaffolding protein that mediates various protein-protein interactions in the synapses. SPIN90 also contains a serine/ threonine-rich region, a potential phosphorylation site. Thus, its interaction with a variety of proteins could be regulated in a activity-dependent manner. Indeed, our data showed that high $\mathrm{KCl}$ stimulation redistributed SPIN90 to the presynaptic terminal. It would be of interest to see whether the effect of SPIN90 on endocytosis could be regulated by phosphorylation/dephosphorylation by certain protein kinases/phosphatases.

\section{References}

Benesch S, Lommel S, Steffen A, Stradal TE, Scaplehorn N, Way M, Wehland J, Rottner K (2002) Phosphatidylinositol 4,5-biphosphate (PIP2)induced vesicle movement depends on N-WASP and involves Nck, WIP, and Grb2. J Biol Chem 277:37771-37776.

Bernard MD, Moschioni M, Napolitani G, Rappuoli R, Montecucco C (2000) The VacA toxin of Helicobacter pylori identifies a new intermediate filament-interacting protein. EMBO J 19:48-56.

Chang S, Camilli PD (2001) Glutamate regulates actin-based motility in axonal filopodia. Nat Neurosci 4:787-793.

Di Paolo G, Pellegrini L, Letinic K, Cestra G, Zoncu R, Voronov S, Chang S, Guo J, Wenk M, De Camilli P (2002) Recruitment and regulation of phosphatidylinositol phosphate kinase type 1 gamma by the FERM domain of talin. Nature 420:85-89.

Fernandez-Alfonso T, Ryan T (2004) The kinetics of synaptic vesicle pool depletion at CNS synaptic terminals. Neuron 41:943-953.

Gad H, Ringstad N, Low P, Kjaerulff O, Gustafsson J, Wenk M, Paolo GD, Nemoto Y, Crun J, Ellisman MH, Camilli PD, Shupliakov O, Brodin L (2000) Fission and uncoating of synaptic clathrin-coated vesicles are perturbed by disruption of interactions with the $\mathrm{SH} 3$ domain of endophilin. Neuron 27:301-312.

Harata N, Pyle J, Aravanis A, Mozhayeva M, Kavalali E, Tsien R (2001) Limited numbers of recycling vesicles in small CNS nerve terminals: implications for neural signaling and vesicular cycling. Trends Neurosci 24:637-643.

Hussain NK, Jenna S, Glogauer M, Quinn CC, Wasiak S, Guipponi M, Antonarakis SE, Kay BK, Stossel TP, Lamarche-Vane N, McPherson PS (2001) Endocytic protein intersectin-l regulates actin assembly via Cdc42 and N-WASP. Nat Cell Biol 3:927-932.

Kessels M, Qualmann B (2002) Syndapins integrate N-WASP in receptormediated endocytosis. EMBO J 21:6083-6094.

Kessels MM, Engqvist-Goldstein AE, Drubin DG, Qualmann B (2001) Mammalian Abp1, a signal-responsive F-actin-binding protein, links the actin cytoskeleton to endocytosis via the GTPase dynamin. J Cell Biol 153:351-366.

Lee E, Camilli PD (2002) Dynamin at actin tails. Proc Natl Acad Sci USA 99:161-166.

Li Z, Murthy VN (2001) Visualizing postendocytic traffic of synaptic vesicles at hippocampal synapses. Neuron 31:593-605.

Lim CS, Park ES, Kim DJ, Song YH, Eom SH, Chun JS, Kim JH, Kim JK, Park D, Song WK (2001) SPIN90 (SH3 Protein Interacting with Nck, $90 \mathrm{kDa}$ ), an adaptor protein that is developmentally regulated during cardiac myocyte differentiation. J Biol Chem 276:12871-12878.

Lim CS, Kim SH, Jung JG, Kim JK, Song WK (2003) Regulation of SPIN90 phosphorylation and interaction with Nck by ERK and cell adhesion. J Biol Chem 278:52116-52123.

McNiven M, Kim L, Krueger E, Orth J, Cao H, Wong T (2000) Regulated interactions between dynamin and the actin-binding protein cortactin modulate cell shape. J Cell Biol 151:187-198.

Merrifield CJ, Feldman ME, Wan L, Almers W (2002) Imaging actin and dynamin recruitment during invagination of single clathrin-coated pits. Nat Cell Biol 4:691-698.

Micheva K, Buchanan J, Holz R, Smith S (2003) Retrograde regulation of synaptic vesicle endocytosis and recycling. Nat Neurosci 6:925-932.

Miki H, Miura K, Matuoka K, Nakata T, Hirokawa N, Orita S, Kaibuchi K, Takai Y, Takenawa T (1994) Association of Ash/Grb-2 with dynamin through the Src homology 3 domain. J Biol Chem 269:5489-5492.

Nikki M, Merilainen J, Lehto V (2002) FAP52 regulates actin organization via binding to filamin. J Biol Chem 277:11432-11440.

Orth JD, McNiven MA (2003) Dynamin at the actin-membrane interface. Curr Opin Cell Biol 15:31-39.

Pfrieger F, Barres B (1997) Synaptic efficacy enhanced by glial cells in vitro. Science 277:1684-1687.

Pyle J, Kavalali E, Piedras-Renteria E, Tsien R (2000) Rapid reuse of readily releasable pool vesicles at hippocampal synapses. Neuron 28:221-231.

Qualmann B, Kelly RB (2000) Syndapin isoforms participate in receptormediated endocytosis and actin organization. J Cell Biol 148:1047-1062.

Qualmann B, Kessels M, Kelly RB (2000) Molecular links between endocytosis and the actin cytoskeleton. J Cell Biol 150:F111-F116.

Ringstad N, Gad H, Low P, Di Paolo G, Brodin L, Shupliakov O, De Camilli P (1999) Endophilin/SH3p4 is required for the transition from early to late stages in clathrin-mediated synaptic vesicle endocytosis. Neuron 24:143-154.

Rohatgi R, Nollau P, Ho HY, Kirschner MW, Mayer BJ (2001) Nck and phosphatidylinositol 4,5-bisphosphate synergistically activate actin polymerization through the N-WASP-Arp2/3 pathway. J Biol Chem 276:26448-26452.

Rozelle AL, Machesky LM, Yamamoto M, Driessens MH, Insall RH, Roth MG, Luby-Phelps K, Marriott G, Hall A, Yin HL (2000) Phosphatidylinositol 4,5-bisphosphate induces actin-based movement of raft-enriched vesicles through WASP-Arp2/3. Curr Biol 10:311-320.

Sankaranarayanan S, Ryan T (2000) Real-time measurements of vesicleSNARE recycling in synapses of the central nervous system. Nat Cell Biol 2:197-204.

Sano K, Hayakawa A, Piao J, Kosaka Y, Nakamura H (2000) Novel SH3 protein encoded by the AF3p21 gene is fused to the mixed lineage leukemia protein in a therapy-related leukemia with $\mathrm{t}(3 ; 11)$ (p21;q23). Blood 95:1066-1068.

Satoh S, Tominaga T (2001) mDia-interacting protein acts downstream of Rho-mDia and modifies Src activation and stress fiber formation. J Biol Chem 276:39290-39294.

Schafer DA (2002) Coupling actin dynamics and membrane dynamics during endocytosis. Curr Opin Cell Biol 14:76-81.

Shupliakov O, Low P, Grabs D, Gad H, Chen H, David C, Takei K, Camilli PD, Brodin L (1997) Synaptic vesicle endocytosis impaired by disruption of dynamin-SH3 domain interactions. Science 276:259-263.

Simpson F, Hussain NK, Qualmann B, Kelly RB, Kay BK, McPherson PS, Schmid SL (1999) SH3-domain-containing proteins function at distinct steps in clathrin-coated vesicle formation. Nat Cell Biol 1:119-124.

Slepnev V, Camilli P (2000) Accessory factors in clathrin-dependent synaptic vesicle endocytosis. Nat Rev Neurosci 1:161-172.

Takei K, Slepnev V, Hauke V, Camilli P (1999) Functional partnership between amphiphysin and dynamic in clathrin-mediated endocytosis. Nat Cell Biol 1:33-39.

Witke W, Podtelejnikov AV, Nardo AD, Sutherland JD, Gurniak CB, Dotti C, Mann M (1998) In mouse brain profilin I and profilin II associate with regulators of the endocytic pathway and actin assembly. EMBO J 17:967-976.

Wu X, Gan B, Yoo Y, Guan J (2005) FAK-mediated Src phosphorylation of endophilin A2 inhibits endocytosis of MT1-MMP and promotes ECM degradation. Dev Cell 9:185-196.

Zakharenko S, Zablow L, Siegelbaum S (2002) Altered presynaptic vesicle release and cycling during mGluR-dependent LTD. Neuron 35:10991110. 